of a novel haemoplasma and vector-borne, zoonotic pathogens indoors. Parasit Vectors. 2015;8:27. http://dx.doi.org/10.1186/ s13071-014-0630-3

8. Stevenson HL, Bai Y, Kosoy MY, Montenieri JA, Lowell JL, Chu MC, et al. Detection of novel Bartonella strains and Yersinia pestis in prairie dogs and their fleas (Siphonaptera: Ceratophyllidae and Pulicidae) using multiplex polymerase chain reaction. J Med Entomol. 2003;40:329-37. http://dx.doi.org/10.1603/ 0022-2585-40.3.329

9. Anda P, Escudero R, Rodriguez-Moreno I, Jado I, Jimenez-Alonso MI. Method and kit for the detection of bacterial species by means of DNA analysis. European patent EP1895015B1. 2012 July 25.

Address for correspondence: Ruth Rodríguez-Pastor, Mitrani Department of Desert Ecology, Jacob Blaustein Institutes for Desert Research, Ben-Gurion University of the Negev, Midreshet Ben-Gurion, 84990, Israel; email: ruth.r.pastor@gmail.com

\section{Mycobacterium bovis Infection in African Wild Dogs, Kruger National Park, South Africa}

\author{
Roxanne L. Higgitt, O. Louis van Schalkwyk, \\ Lin-Mari de Klerk-Lorist, Peter E. Buss, \\ Peter Caldwell, Leana Rossouw, \\ Tebogo Manamela, Guy A. Hausler, \\ Jennie Hewlett, ${ }^{1}$ Emily P. Mitchell, ${ }^{1}$ \\ Paul D. van Helden, Sven D.C. Parsons, ${ }^{2}$ \\ Michele A. Miller ${ }^{2}$
}

Author affiliations: Stellenbosch University, Cape Town, South Africa (R.L. Higgitt, G.A. Hausler, P.D. van Helden, S.D.C. Parsons, M.A. Miller); Department of Agriculture, Forestry and Fisheries, Skukuza, South Africa (O.L. van Schalkwyk, L.-M. de Klerk-Lorist); South African National Parks, Skukuza (P.E. Buss, L. Rossouw, T. Manamela, J. Hewlett); Old Chapel Veterinary Clinic, Pretoria, South Africa (P. Caldwell); National Zoological Gardens of South Africa, Pretoria (E.P. Mitchell)

DOI: https://doi.org/10.3201/eid2507.181653

${ }^{1}$ Current affiliation: University of Pretoria, Onderstepoort, South Africa.

${ }^{2}$ These authors were co-principal investigators for this article.
We screened African wild dogs (Lycaon pictus) in Kruger National Park, South Africa, for Mycobacterium bovis infection using an interferon-gamma release assay. We detected M. bovis sensitization in 20 of 21 packs; overall apparent infection prevalence was $83 \%$. These animals experience high infection pressure, which may affect long-term survival and conservation strategies.

$\mathrm{T}$ he African wild dog (Lycaon pictus) is an endangered carnivore occurring in fragmented, small populations (in South Africa, $<500$ animals). These factors make them susceptible to adverse factors, such as infectious diseases, that may threaten their long-term survival $(1,2)$. Of particular concern are diseases caused by multihost pathogens that are capable of persisting in reservoir host species, such as Mycobacterium bovis, the causative agent of bovine tuberculosis (bTB). This pathogen may pose a major threat to the conservation of endangered host populations (3).

Since 2012, sporadic cases of wild dogs with macroscopic and histological lesions consistent with tuberculosis (TB) have been recorded in South Africa, specifically in Kruger National Park (KNP; $\mathrm{n}=8$ ), uMkuze Game Reserve $(\mathrm{n}=1)$, and Hluhluwe-iMfolozi Park (HiP; $\mathrm{n}=2)$. $M$. bovis infection is endemic in these parks and occurs in multiple species that are preyed upon by wild dogs, such as warthogs, which have an estimated $M$. bovis seroprevalence up to $38 \%$ in KNP $(4,5)$. In 2 cases from KNP, acid-fast bacilli were associated with granulomatous lymphadenitis, and spoligotype analysis of $M$. bovis isolates from lesions in affected wild dogs from KNP (strain type SB0121) and HiP (strain type SB0130) were the same as those found in local prey (6).

$M$. bovis is a novel pathogen of wild dogs; understanding the impact of bTB disease in wild dogs is imperative to making informed management decisions regarding these animals' conservation. Estimation of prevalence would provide a starting point for this investigation but requires diagnostic tools for accurate detection of $M$. bovis infection. To estimate prevalence in the KNP wild dog population, we assessed sensitization to TB antigens ESAT- 6 and CFP-10.

During July 2016-January 2018, we tested blood samples from 77 wild dogs from KNP using an interferongamma release assay (IGRA) developed by our group (7). We tested animals from 21 wild dog packs; 20 of these included $\geq 1$ IGRA-positive animal, indicating widespread exposure to $M$. bovis throughout KNP (Figure). We observed no significant difference in IGRA results based on sex ( $p=0.79$ by 2 -tailed Mann-Whitney test). Overall, the apparent prevalence of $M$. bovis infection was $82 \%(63 / 77$; $95 \%$ CI $72 \%-89 \%$ by modified Wald test).

Few reports of active bTB disease and related deaths have been documented in wild dogs, so the high apparent 
prevalence of $M$. bovis infection in the KNP population is surprising. However, similarly high prevalence estimates have been reported for African lions (Panthera leo leo) in KNP using the tuberculin skin test $(55 \%$; $95 \%$ CI $45 \%-$ $65 \%)(8)$ and a cytokine gene expression assay (44\%; $95 \%$ CI $32 \%-57 \%$ ) (9). These results highlight the high infection pressure for carnivores within KNP.

The prevalence estimate in wild dogs was based on detection of immune sensitization to $M$. bovis-specific antigens. Although the infection status of a small number of animals was confirmed by antemortem mycobacterial culture of oropharyngeal swabs (4 $[7.0 \%]$ of 57 wild dogs tested), we could not confirm the infection status of all of

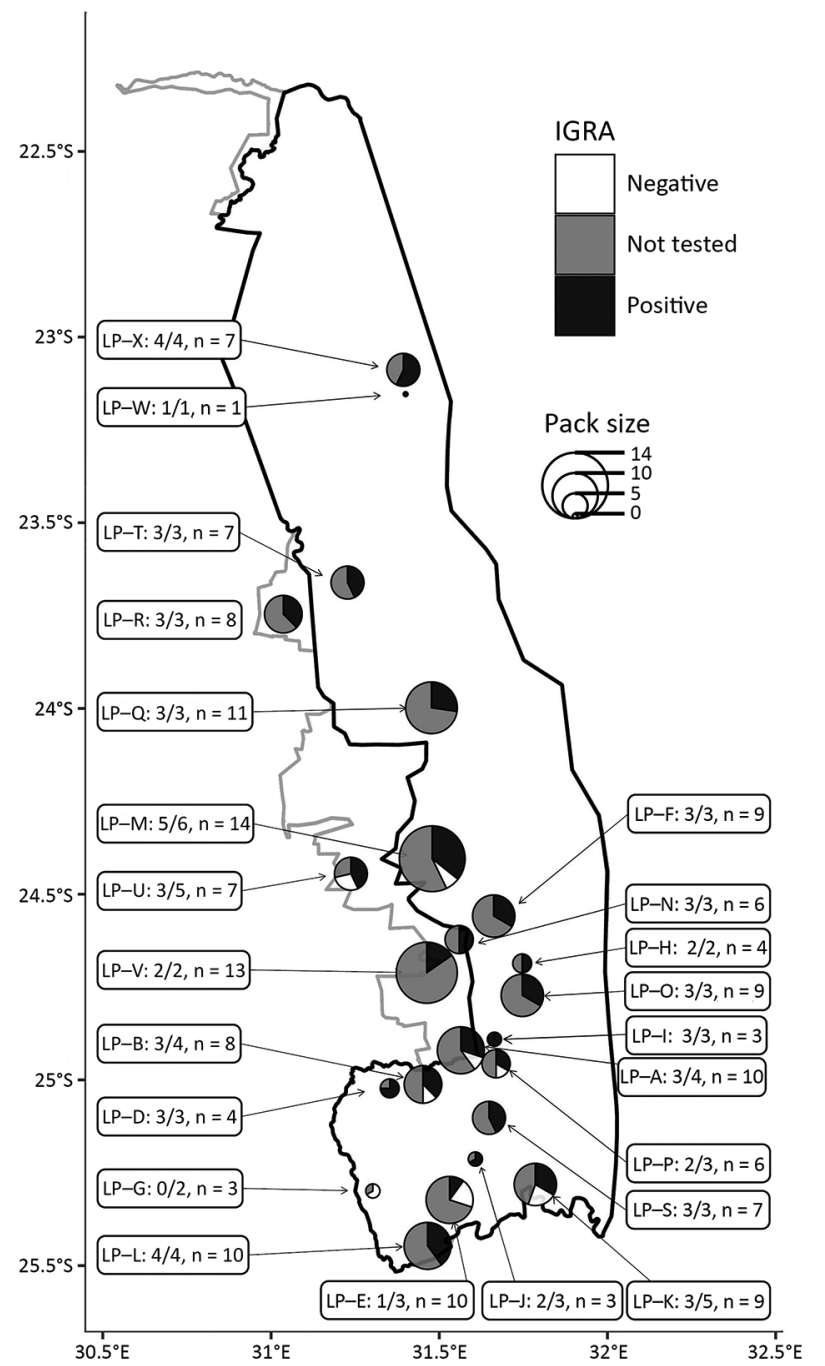

Figure. Mycobacterium bovis infection in African wild dog packs, Kruger National Park, South Africa. Each pie chart indicates the position of a pack at time of sampling; the size of the pack ( $\mathrm{n}$ value); and the proportion of test-positive, test-negative, and untested animals. The pack name (e.g., LP-A) and the proportion of tested animals that were test-positive (e.g., 2/2) are shown. A single wild dog that was not part of a pack was included. IGRA, interferon-gamma release assay. the animals included in this study. Furthermore, during the time of the study, most animals included in this analysis appeared to be healthy. Therefore, further investigations will be required to clarify the progression of $M$. bovis infection and risk of bTB disease in this species.

These results have implications for managing the wild dog metapopulation in South Africa, which involves translocation of animals across the country to maintain genetic diversity and to achieve conservation goals (2). The risk of introducing $M$. bovis into previously uninfected areas by an infected wild dog is unknown, and studies on transmission will be crucial in assessing this risk.

Survival of a species is affected by a complex array of factors, of which disease is only one. Currently, the KNP wild dog population appears to be stable (2), despite the apparent high prevalence of M. bovis infection. Favorable conditions, such as abundance of prey, may support high reproductive rates. However, with environmental changes, such as prolonged drought, the vulnerability of host populations to infectious disease may be more pronounced (10).

In conclusion, this study shows widespread exposure of wild dog packs to M. bovis in KNP, with high infection pressure to individual dogs. Although the impact of disease on population numbers is unknown, wild dogs infected with $M$. bovis, even young animals, have been observed to have generalized disease leading to death. Further investigations into the progression of $M$. bovis infection, the risk for transmission, and the probability of developing progressive disease are needed to assess the threat of this emerging disease in African wild dog populations.

\section{Acknowledgments}

We acknowledge the contributions made by the veterinary technicians of the Kruger National Park State Veterinary Office in Skukuza, South Africa, as well as David Cooper, Birgit Eggers, and Ezemvelo KZN Wildlife for assistance with wild dog sampling and data collection.

Research protocols were approved by the University of Stellenbosch Animal Care and Use Committee (SU-ACUD16-00076) and the Department of Agriculture, Forestry and Fisheries, South Africa (12/11/1/7/2). This research was funded (partially or fully) by the South African government through the South African Medical Research Council and the National Research Foundation of South Africa (grant no. 86949).

\section{About the Author}

Ms. Higgitt is a research assistant at the Department of Biomedical Sciences, Stellenbosch University. Her current research interests are understanding immune responses of South Africa carnivores to $M$. bovis infection and the development of diagnostic assays for these species. 


\section{References}

1. Woodroffe R, Ginsberg J, Macdonald D. Status survey and conservation action plan: the African wild dog. Gland (Switzerland): International Union for Conservation of Nature/ Species Survival Commission Canid Specialist Group; 1997.

2. Davies-Mostert H, Page-Nicholson S, Marneweck D, Marnewick K, Cilliers D, Whittington-Jones B, et al. A conservation assessment of Lycaon pictus. In: Child M, Roxburgh L, Do Lihn San E, Raimondo D, Davies-Mostert H, editors. The red list of mammals of South Africa, Swaziland and Lesotho. Pretoria (South Africa): South African National Biodiversity Institute and Endangered Wildlife Trust; 2016 [cited 2018 Aug 20]. https://pdfs.semanticscholar.org/7259/ e724f3111c2e68609d41cb630f91692f1892.pdf

3. Woodroffe R. Managing disease threats to wild mammals. Anim Conserv. 1999;2:185-93. http://dx.doi.org/10.1111/ j.1469-1795. 1999.tb00064.x

4. Renwick AR, White PCL, Bengis RG. Bovine tuberculosis in southern African wildlife: a multi-species host-pathogen system. Epidemiol Infect. 2007;135:529-40. http://dx.doi.org/10.1017/ S0950268806007205

5. Roos EO, Olea-Popelka F, Buss P, de Klerk-Lorist L-M, Cooper D, van Helden PD, et al. Seroprevalence of Mycobacterium bovis infection in warthogs (Phacochoerus africanus) in bovine tuberculosis-endemic regions of South Africa. Transbound Emerg Dis. 2018;65:1182-9. http://dx.doi.org/10.1111/ tbed. 12856

6. Michel AL, Coetzee ML, Keet DF, Maré L, Warren R, Cooper D, et al. Molecular epidemiology of Mycobacterium bovis isolates from free-ranging wildlife in South African game reserves. Vet Microbiol. 2009;133:335-43. http://dx.doi.org/10.1016/ j.vetmic.2008.07.023

7. Higgitt RL, van Schalkwyk OL, deKlerk-Lorist LM, Buss PE, Caldwell P, Rossouw L, et al. An interferon gamma release assay for the detection of immune sensitization to Mycobacterium bovis in African wild dogs (Lycaon pictus). J Wildl Dis. 2018:55. http://dx.doi.org/10.7589/2018-03089

8. Keet D, Davies-Mostert H, Bengis R, Funston P, Buss P, Hofmeyr M, et al. Disease risk assessment workshop report: African lion (Panthera leo) bovine tuberculosis. Conservation Breeding Specialist Group/CBSG Southern Africa, Endangered Wildlife Trust. 2009 [cited 2018 Aug 20]. http://www.wcs-ahead. org/gltfca_grants/pdfs/lion_tb_risk_report_final.pdf

9. Sylvester TT, Martin LER, Buss P, Loxton AG, Hausler GA, Rossouw L, et al. Prevalence and risk factors for Mycobacterium bovis infection in African lions (Panthera leo) in the Kruger National Park. J Wildl Dis. 2017;53:372-6. http://dx.doi.org/10.7589/2016-07-159

10. de La Rocque S, Rioux JA, Slingenbergh J. Climate change: effects on animal disease systems and implications for surveillance and control. Rev Sci Tech. 2008;27:339-54.

Address for correspondence: Michele A. Miller, Stellenbosch University DST-NRF Centre of Excellence for Biomedical TB Research/MRC Centre for Tuberculosis Research/Division of Molecular Biology and Human Genetics, Faculty of Medicine and Health Sciences, PO Box 241, Cape Town 8000, South Africa; email: miller@sun.ac.za

\section{Identification of Internationally Disseminated Ceftriaxone-Resistant Neisseria gonorrhoeae Strain FC428, China}

\author{
Shao-Chun Chen, Yan Han, Liu-Feng Yuan, \\ Xiao-Yu Zhu, Yue-Ping Yin
}

Author affiliations: Chinese Academy of Medical Sciences and Peking Union Medical College, Nanjing, China (S.-C. Chen, Y. Han, X.-Y. Zhu, Y.-P. Yin); Chinese Center for Disease Control and Prevention, Nanjing (S.-C. Chen, Y. Han, X-.Y. Zhu, Y.-P. Yin); Beijing Ditan Hospital Capital Medical University, Beijing, China (L.-F. Yuan)

DOI: https://doi.org/10.3201/eid2507.190172

In 2016, we identified a ceftriaxone-resistant Neisseria gonorrhoeae isolate in China. The strain genotype was identical to the resistant clone FC428 that originated in Japan. Enhanced international collaborative surveillance programs are crucial to track the transmission of the ceftriaxone-resistant clones.

O eftriaxone has been used to treat gonorrhea in China and most other countries for $>1$ decade, but the level of decreased susceptibility or clinical resistance to ceftriaxone has increased (1). Moreover, the international spread of ceftriaxone-resistant clones has been recognized as a threat to effective control of gonorrhea (2). We describe an imported ceftriaxone-resistant $N$. gonorrhoeae strain isolated in China in 2016.

The patient was a heterosexual man in his late twenties. He reported unprotected 1-night heterosexual sex in Beijing in July 2016. Urethral discharge with dysuria occurred 3 days after the sexual activity. He was prescribed oral cephalosporin when he visited a private clinic in July. Because the urethral discharge did not resolve, he visited the sexually transmitted diseases clinic in Beijing Ditan Hospital (Beijing, China) in August.

Laboratory analysis of a urethral swab sample found gram-negative diplococci within leukocytes. Culture and nucleic acid amplification test were positive for $N$. gonorrhoeae. Screening for other sexually transmitted infections by nucleic acid amplification test was negative for Chlamydia trachomatis, Ureaplasma urealyticum, and Mycoplasma hominis.

The patient was treated with a 1-g intravenous dose of ceftriaxone once per day for 3 days. His symptoms improved after 3 days, and a test-of-cure by culture showed 